\title{
Review of: "Dithiothreitol causes toxicity in C. elegans by modulating the methionine-homocysteine cycle"
}

Muneyoshi Kanai

Potential competing interests: The author(s) declared that no potential competing interests exist.

The authors elucidated that DTT toxicity was modulated by the bacterial diet, especially vitamin B12, and methionine synthase-dependent manner. Moreover, by using a forward genetic screen, authors identified a gene that contribute to DTT resistance. Finally, authors clarified the role of SAM per se and SAM-dependent methyltransferase in DTT resistance. These results would be meaningful for gaining basic knowledge academically and technology development related to pathomechanism in this report. Accordingly, I consider this MS as acceptable to be published in bioRxiv article. However, in order to be accepted as this journal, there are some points that need correction and confirmation.

P4L26-29

“The amount of dietary vitamin B12 available to C. elegans can be reliably determined by a dietary sensor strain expressing green fluorescent protein (GFP) under the promoter of acyl-coenzyme A dehydrogenase (acdh-1) gene"

Can impacts on DTT resistance by a bacterial diet-dependent manner be explained by the effects of vitamin B12 alone? What about the effects of other ingredients?

P8L26-27

"these results suggested that SAM depletion is the primary cause of DTT toxicity." Isn't it necessary to perform SAM addition test and quantify of intracellular SAM in DTT resistance? 